\title{
Electrophysiological Evidence for Early Contextual Influences during Spoken-Word Recognition: N200 Versus N400 Effects
}

\author{
Daniëlle van den Brink, Colin M. Brown, and Peter Hagoort
}

\begin{abstract}
An event-related brain potential experiment was carried out to investigate the time course of contextual influences on spoken-word recognition. Subjects were presented with spoken sentences that ended with a word that was either (a) congruent, (b) semantically anomalous, but beginning with the same initial phonemes as the congruent completion, or (c) semantically anomalous beginning with phonemes that differed from the congruent completion. In addition to
\end{abstract}

\section{INTRODUCTION}

In everyday speech, people hear words in the context of other words, usually in the form of sentences. In the literature on language comprehension, there is evidence to suggest that contextual influences play a role in the on-line recognition of spoken words (Salasoo \& Pisoni, 1985; Marslen-Wilson \& Tyler, 1980; Morton, 1969). In the recognition of words spoken in isolation, a number of spoken-word recognition models converge on the idea that multiple lexical candidates that share word onset are accessed on the basis of an analysis of the initial phoneme(s) of a word (McQueen, Norris, \& Cutler, 1994; Norris, 1994; Norris, McQueen, \& Cutler, 1995; Goldinger, Luce, \& Pisoni, 1989; Goldinger, Luce, Pisoni, \& Marcario, 1992; Marslen-Wilson, 1987; MarslenWilson \& Welsh, 1978; McClelland \& Elman, 1986). As pronunciation of a word progresses over time, lexical candidates are dropped as soon as they no longer correspond to the incoming acoustic signal. Selection of the proper candidate is said to take place when only one candidate is left that still matches the acoustic signal.

Word recognition in sentences additionally requires that the selected word is integrated into a higher-order meaning representation of the sentence context. The impact of sentential-contextual information on the recognition process is a matter of debate, with several

Max Planck Institute for Psycholinguistics, Nijmegen, The Netherlands finding an N400 effect in the two semantically anomalous conditions, we obtained an early negative effect in the semantically anomalous condition where word onset differed from that of the congruent completions. It was concluded that the N200 effect is related to the lexical selection process, where word-form information resulting from an initial phonological analysis and content information derived from the context interact.

models assuming a high degree of interactivity between contextual and acoustic information, and other models assigning priority to the acoustic analysis. A key issue here concerns the relative moment in time at which context starts to exert an effect on word recognition. The present study was designed to investigate the time course of contextual effects on spoken-word recognition. The focus of our study is on the electrophysiological manifestation of these effects, and less on the empirical separation of competing word recognition models. We seek to validate an early electrophysiological manifestation of the processing interface between words and their context, which will provide a real-time measure with which to test competing spoken-word recognition models.

Event-related potentials (ERPs) reflect the sum of simultaneous postsynaptic activity of a large number of neurons recorded at the scalp as small voltage fluctuations in the electroencephalogram. A central finding in the ERP literature on language is a negative-going component that typically peaks at $400 \mathrm{msec}$ after stimulus onset, the N400 (cf. Kutas \& Hillyard, 1980). The N400 component is related to semantic processing of the eliciting word and is observed in both the visual and the auditory modality. Although N400 effects have sometimes been related to lexical access (Kutas \& Federmeier, 2000), today the most widely held view is that in sentence contexts, the N400 amplitude indexes the relative ease of semantic integration (e.g., Brown \& Hagoort, 1993, 2000; Holcomb, 1993): Words that are incongruent or less fitting given the preceding sentence 
frame typically elicit a much larger N400 than words that fit well within the context. This N400 effect has a posterior scalp distribution and an onset around 200250 msec after word onset (for reviews, see Osterhout \& Holcomb, 1995; Kutas \& Van Petten, 1988, 1994). However, semantic integration of the perceived word into the sentence context is one of the last subprocesses in spoken-word recognition. If the N400 effect solely reflects the semantic integration process, is there any other evidence in the electrophysiological signal that context exerts an influence prior to semantic integration? Or does the N400 effect not only reflect integration processes, but also early semantic processing at a level where lexical and contextual information interact? A number of recent ERP studies on spoken-word processing have addressed these issues.

Connolly and Phillips (1994) carried out a study to investigate the possibility of the existence of a separate ERP component in the auditory modality, preceding the $\mathrm{N} 400$, that, in their terminology, might reflect some kind of "preprocessing phase" related to contextual influences. In earlier studies, Connolly, Stewart, and Phillips (1990) and Connolly, Phillips, Stewart, and Brake (1992) had found a negativity peaking at $200 \mathrm{msec}$. In their 1994 study, they hypothesized that this N200 was related to a phonological process that assessed whether the initial sounds of a word fit the sentential context. On the basis of the preceding context a semantic expectation of a particular word is formed, and if the initial phonemes of the perceived word do not match the initial phonemes of the expected word, an N200 is elicited. The underlying idea is that, certainly in the auditory modality, the semantic system could benefit from such an early process. To test their account of an early negativity reflecting a phonological process, Connolly and Phillips (1994) included four conditions in their design. The experimental items consisted of medium to high constraining sentences that ended with a semantically correct or anomalous word. The semantically correct sentencefinal words were either the highest-cloze probability words and therefore the expected endings (e.g., "The piano was out of tune) or words that were of a lower cloze probability (e.g., "Don caught the ball with his glove," where hand would have been expected). ${ }^{1}$ The semantically anomalous sentence-final words began with phonemes that were either similar to those of the expected word (e.g., "The gambler had a streak of bad luggage," where luck would have been expected) or different (e.g., "The dog chased the cat up the queen," where tree would have been expected).

In the two conditions where the onset of the word did not match that of the highest-cloze probability word, Connolly and Phillips (1994) obtained an early negativity (now peaking between 270 and $300 \mathrm{msec}$ ). They did not find an early negativity in the remaining two conditions, not even in the semantically anomalous condition where word onset was identical to the highest-cloze probability word, and where they did obtain an N400. Therefore, Connolly and Phillips claimed that the early negativity was a phonological mismatch negativity (PMN), reflecting a discrepancy between the initial phonemes of the expected word and the actually perceived word. Their results seem to support the notion that a semantic expectation of a particular word is formed on the basis of the preceding context. Subsequently, the incoming signal is matched against the phonemic template of the expected word. If the initial phonemes do not match, a PMN is elicited.

Van Petten, Coulson, Rubin, Plante, and Parks (1999) performed a study somewhat similar to that of Connolly and Phillips (1994). In this study, they made use of information about the isolation points (IP) of words, namely, the minimum amount of acoustic signal necessary to identify a spoken word in the absence of contextual information (cf. Grosjean, 1980). The IPs were established with the gating technique, in which subjects hear increasingly longer fragments of a word, and report after each fragment which word they think is being presented. Van Petten et al. hypothesized that "... if semantic processes begin to operate on the partial and incomplete results of perceptual analyses, then the influence of semantic context might begin before the IP, as soon as the acoustic input becomes discrepant with semantic expectations" (p. 397). Their experimental items consisted of high to low constraining sentences that ended either (a) with the highestcloze probability word, (b) with a word that rhymed with the highest-cloze probability word, (c) with a semantically anomalous word that shared the same initial phonemes as the highest-cloze probability word, or (d) with an anomalous word of which the initial phonemes differed from those of the highest-cloze probability word. For example: "It was a pleasant surprise to find that the car repair bill was only seventeen dollars/scholars/dolphins/bureaus."

The results revealed that the sentence-final words in the three anomalous conditions all elicited a significantly larger N400 than the high-cloze probability endings. Moreover, the onset of the N400 effect differed between conditions. In the fully anomalous and rhyme conditions, the onset of the N400 effect preceded the IP of the sentence-final words. Relative to these two conditions, the onset of the N400 effect in the condition where the anomalous word shared its phonemic onset with the highest-cloze probability word was found to be delayed by some $200 \mathrm{msec}$ and corresponded to the IP. It was concluded that the onset of the N400 effect was related to the moment at which the acoustic input first diverged from semantic expectation. Van Petten et al. (1999) claimed that the N400 effect reflects the semantic incompatibility between the semantic expectation that is derived from the sentence context and the meaning of the acoustic word input. This account assumes that the incoming acoustic in- 
formation is semantically analyzed and contrasted with the semantic expectation. This aspect was not further elaborated in terms of specific lexical representations, or more general semantic expectations. Van Petten et al. concluded that the N400 effect reflects semantic processing of the perceived word and that this semantic processing begins on partial and incomplete information about the perceived word.

The different results obtained in these studies, that is, an early negativity versus an early onset of the N400 effect, resulted in different interpretations. The finding of an early negativity led Connolly and Phillips (1994) to conclude that on the basis of the preceding context a specific phonological expectation is formed. The early onset of the N400 effect in Van Petten et al.'s study led them to conclude that a semantic expectation is formed. Despite these contrasting results, both studies provide evidence for early contextual influences during word recognition. However, neither a phonological nor a semantic expectation account necessarily implies that context already influences word recognition prior to lexical access. Suppose that, following the Shortlist model (Norris, 1994), contextual influences arise only after the bottom-up activation of a number of lexical candidates on the basis of the initial phonemes of the perceived word. If the contextual specifications do not support any of the lexical candidates in the set (e.g., on the basis of their semantic features), a congruency effect occurs, showing up either as an N400 effect (Van Petten et al., 1999) or as an early negativity (Connolly \& Phillips, 1994).

This account fits with how Hagoort and Brown (2000) interpreted their results of a recent ERP study. The authors presented their subjects with spoken sentences that ended with a semantically congruent or anomalous word that did not have the same phonemic onset as the congruent completions. A biphasic negative shift was observed to the semantically anomalous endings. This biphasic shift was composed of two effects, an early effect that peaked around 250 msec (the N250 effect) and the N400 effect. The authors suggested that the N250 effect occurs whenever "... the contextual specifications do not support the form-based activation of a lexical candidate ..." (p. 1528).

The fact that these studies yield contrasting results (early negativity vs. early onset of the N400 effect) remains somewhat surprising, and a few remarks are in order. The grand average waveforms of Connolly and Phillips (1994) are rather noisy, and identification of separate components is therefore difficult. On the other hand, although Van Petten et al. (1999) claim not to have found an early negativity, inspection of the waveforms of their Experiment 3 does reveal the possibility of an early negativity, at least in the rhyme and fully anomalous conditions. In itself, this finding would not harm their account, but it could add evidence to the existence of a separate early negativity.
The issue of the exact time course of contextual influences on spoken-word recognition is important for our understanding of the underlying cognitive architecture of the language system. ERP research allows us to investigate this underlying cognitive architecture through identification of electrophysiological correlates of spoken-word recognition processes. The three studies mentioned above indicate that context has an early effect on spoken-word recognition. The present study follows on from these studies and was designed to give a better insight into this early context effect.

The time course of contextual influences on spokenword recognition was evaluated with ERPs. Taking into account the possibility of some delay between underlying processes and their manifestation in the ERP waveform, the latest moment at which context starts to exert its influence was assumed to be revealed as the onset of a congruity effect in the waveforms, as indicated by the divergence between a congruent and an incongruent condition. We used semantically constraining Dutch sentences with sentence-final words that differed across three conditions. In the fully congruent (FC) condition, sentences ended with the highest-cloze probability word for that sentence: "De schilder kleurde de details in met een klein penseel" ("The painter colored the details with a small paint brush"). The other two conditions both ended anomalously, but the point at which the sentence-final words became incongruent differed between these two conditions. The completions of the sentences in the initially congruent (IC) condition began with the same phonemes as the highest-cloze probability words in the FC completions: "De schilder kleurde de details in met een klein pensioen" ("The painter colored the details with a small pension"). In contrast, the fully incongruent (FI) sentence-final words had initial phonemes that differed from the highest-cloze probability word: "De schilder kleurde de details in met een klein doolbof" ("The painter colored the details with a small labyrinth"). We hypothesized that the onset of the congruity effect between the FC and FI conditions should precede the onset of the congruity effect between the FC and IC conditions. We were also curious to see how this congruity effect would manifest itself; as the onset of a monophasic N400 effect as in the Van Petten et al. study (1999), or as a biphasic negative shift consisting of an early negative shift and an N400 effect as in the Connolly and Phillips study (1994) and the Hagoort and Brown study (2000).

We also investigated ERP effects as a function of the moment at which the sentence-final words in the FC and IC conditions started to acoustically diverge from each other. This moment was labeled the divergence point. We were interested in whether the manifestation of the congruity effect based on word onset (FC vs. FI) would differ from the congruity effect based on the divergence point (FC vs. IC). This line of investigation provides an additional perspective on the validity of the distinction 


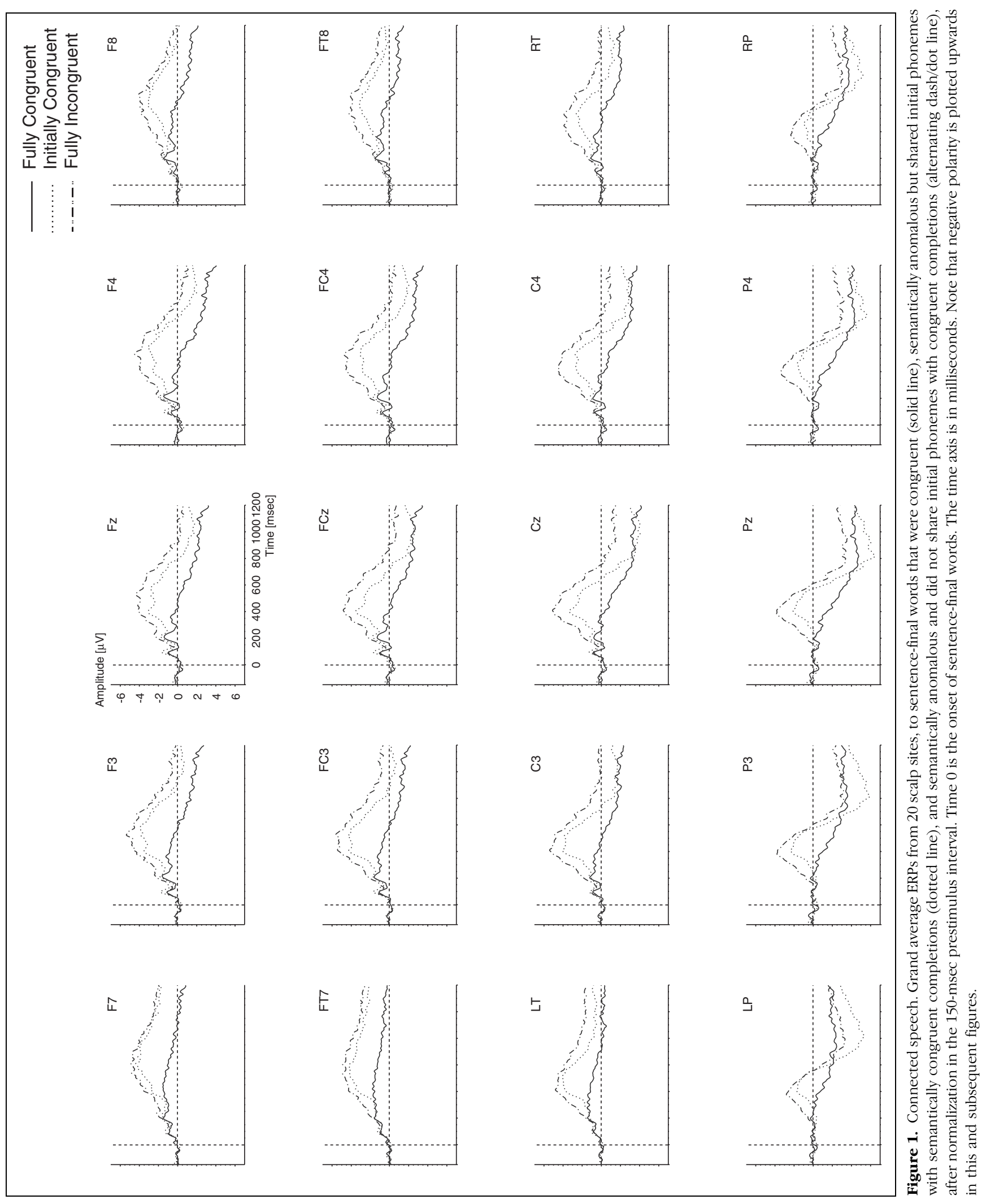


between an early negative shift and the N400 effect during spoken-word recognition.

\section{RESULTS}

The data of five subjects were not analyzed: The data of one subject were lost because of a computer malfunction and the data of four others showed excessive alpha activity (a pronounced $10-\mathrm{Hz}$ rhythm throughout the waveforms). As this study was designed to investigate the possible existence of a relatively small and short-lived early negativity, we were concerned that excessive alpha would distort the signals in this early latency window to such an extent that it would influence the results. For the remaining 21 subjects, data from critical trials were analyzed according to the following procedure. Prior to off-line averaging, all single-trial waveforms were screened for eye movements, electrode drifting, amplifier blocking, and EMG artifacts in a critical window that ranged from $150 \mathrm{msec}$ before onset of the sentence-final word to $1900 \mathrm{msec}$ after onset of the sentence-final word. Trials containing artifacts were rejected (12.8\% in FC, $13.4 \%$ in IC, and $11.8 \%$ in FI). For each subject, average waveforms were computed across all remaining trials per condition after normalizing the waveforms of the individual trials on the basis of the averaged activity of $150 \mathrm{msec}$ before onset of the sentence-final word. This is a standard baselining procedure whereby the average of the 150msec epoch is subtracted from each subsequent time point in the waveform.

Figure 1 displays the grand average waveforms by electrode site time-locked to the onset of the sentencefinal word. There are several things to note. First, in all three conditions, the sentence-final words elicited an N100 component. This component appears to be larger (but is in fact not) in the IC condition than in the FC and FI conditions. This is most visible in the frontal to central electrodes. ${ }^{2}$ It is not common to find a clear N100 component in a study using natural connected speech, given the continuous nature of the speech signal (Connolly et al., 1990, 1992, 1994). However, in our study, all sentence-final words began with a plosive, which is a relatively clear onset marker in connected speech. In addition, the homogeneity of the sentencefinal word onsets presumably added to the clear presence of the N100 component in the averaged waveforms. Second, a negativity at approximately $200 \mathrm{msec}$ is visible in the waveforms. This negativity is apparent in all three conditions, but is largest in the FI condition and is most visible over the frontal sites (see also Figure 2). Third, the semantically anomalous sentence-final words in the IC and FI conditions elicit a broad negativity peaking at approximately $400 \mathrm{msec}$, which is more negative than the ERP elicited in the FC condition. In turn, this broad negativity is larger in the FI condition than in the IC condition. Its latency characteristics and morphology are similar to previously reported N400 effects. Finally, Figure 1 shows a late positivity between 600 and $1000 \mathrm{msec}$, which is largest in the IC condition. This late positivity reaches maximal amplitude at around $800 \mathrm{msec}$ and is maximal over parietal sites. In the literature, there are indications that late positivities reflect wrap-up processes at the end of sentences (cf. Juottonen, Revonsuo, \& Lang, 1996).

Statistical analyses of the congruity effects consisted of a number of repeated measures analyses of variance (ANOVAs) with mean amplitude values computed for each subject and each electrode in different latency windows: (a) 150-250 msec after critical-word onset for the early negativity (N200), (b) 300-500 msec after final-word onset for the N400, and (c) 600-1000 msec after final-word onset for the late positivity. The latency windows were determined after careful visual inspection of the grand average waveforms. The latency window for the early negativity corresponds to the onset of the ascending flank and the offset of the descending flank. The N400 latency window corresponds to the onset of the ascending flank and 100 msec after its maximal amplitude. This 300- to 500msec interval is a standard time window for measuring N400 effects. The latency window for the late positivity corresponds to the onset of the ascending flank and 200 msec after its maximal amplitude. For each latency window, the results were first analyzed in an omnibus ANOVA that crossed all three levels of the congruity

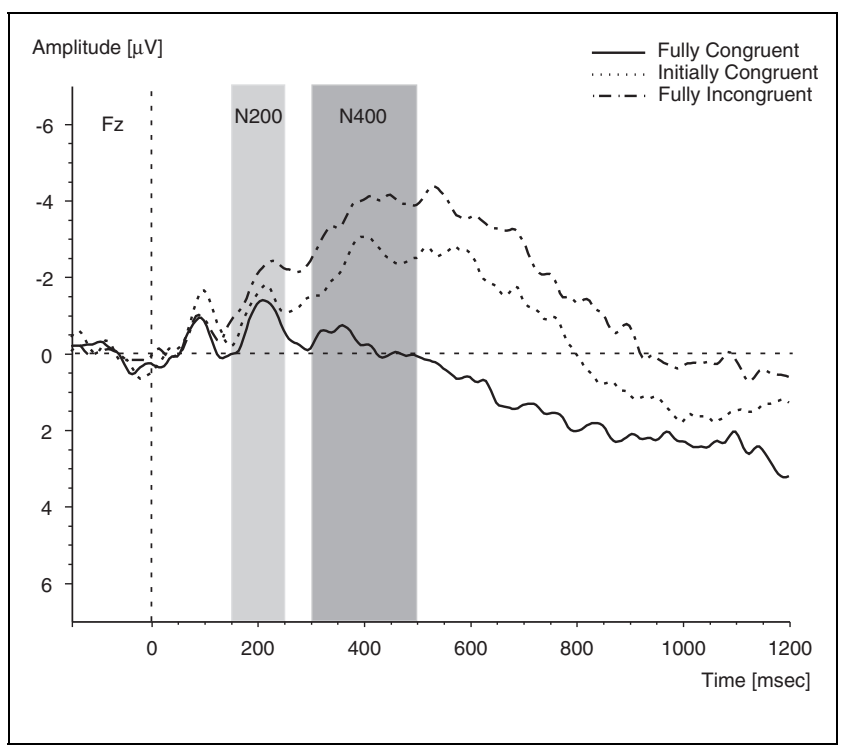

Figure 2. Connected speech. Grand average ERPs from the Fz electrode, to sentence-final words that were congruent (solid line), semantically anomalous but shared initial phonemes with semantically congruent completions (dotted line), and semantically anomalous and did not share initial phonemes with congruent completions (alternating dash/dot line), after normalization in the $150-\mathrm{msec}$ prestimulus interval. Time 0 is the onset of sentence-final words. The time axis is in milliseconds. 
factor (FC, IC, and FI) with the 29-level electrode factor. In addition to the omnibus ANOVA, a priori pairwise comparisons between the congruity conditions were tested using ANOVAs with a 2-level congruity factor. For the N200 latency window, we expected the ERP in the FC condition (baseline) to differ in amplitude from the ERP in the FI condition (FC vs. FI), but not from the one in the IC condition (FC vs. IC). In the N400 latency window, as well as the late positivity latency window, we expected the ERP in the FC condition to differ in amplitude from the ERPs in both the FI (FC vs. FI) and the IC condition (FC vs. IC), but these latter were not expected to differ from each other (FI vs. IC). Scalp distributions of the congruity effects were subsequently explored in three separate ANOVAs: an ANOVA on anterior versus posterior, with a site (anterior/posterior) by electrode design (anterior: Fz, FCz, F3, F4, F7, F8, FC3, FC4, FT7, FT8; posterior: Cz, Pz, CP3, CP4, LTP, RTP, P3, P4, LP, RP); anterior left versus anterior right, with a site (left/right hemisphere) by electrode design (anterior left: AF3, F3, F7, FC3, FT7; anterior right: AF4, F4, F8, FC4, FT8); posterior left versus posterior right, with a site (left/right hemisphere) by electrode design (posterior left: CP3, LTP, P3, LP, PO7; posterior right: CP4, RTP, P4, RP, PO8). Univariate $F$ tests with more than one degree of freedom in the numerator were adjusted by means of the Greenhouse-Geisser/Box's epsilon hat correction (Maxwell \& Delaney, 1989). The original degrees of freedom and adjusted $p$ values will be reported.

\section{The Early Negativity/N200 Latency Window: 150-250 msec}

Table 1 displays the results of the mean ERP amplitude ANOVAs in the 150-250-msec latency range. The omnibus ANOVA for the N200 latency window resulted in a significant main effect of congruity. The a priori pairwise comparisons revealed that the FI completions elicited a larger N200 than the FC completions (corresponding to an effect of $0.71 \mu \mathrm{V}$ ), and that the ERPs in the FC and IC conditions did not significantly differ in amplitude (a difference of $0.17 \mu \mathrm{V})$. In these pairwise analyses, none of the interactions of congruity with electrodes reached significance. Topographical analyses of the FC versus FI condition revealed no significant interactions of congruity with site.

\section{The N400 Latency Window: 300-500 msec}

Table 2 displays the results of the mean ERP amplitude ANOVAs in the 300-500-msec latency range. The omnibus ANOVA indicated that the basic congruity effect in the N400 latency window was robust. Further analysis revealed that both the FI and the IC completions elicited a larger $\mathrm{N} 400$ than the FC completions (corresponding to effects of 3.36 and $1.97 \mu \mathrm{V}$, respectively). In addition, the FI and the IC conditions differed significantly from each other in the N400 latency window (corresponding to a $1.38-\mu \mathrm{V}$ average amplitude difference), and an interaction with electrodes was found as well. Topographical analyses showed that the congruity effects in relation to the

Table 1. ANOVA on Mean ERP Amplitude in the 150-250-msec Latency Range (N200)

\begin{tabular}{|c|c|c|c|c|c|}
\hline & Source & $d f$ & $F$ & $M S E$ & $p$ \\
\hline \multicolumn{6}{|c|}{ Omnibus ANOVA (29 electrodes) } \\
\hline \multirow[t]{2}{*}{ Overall } & Con & 2,40 & 4.25 & 84.18 & $.023^{*}$ \\
\hline & Con $\times \mathrm{El}$ & 56,1120 & 1.98 & 0.59 & .075 \\
\hline \multirow[t]{2}{*}{ FC vs. FI } & Con & 1,20 & 6.90 & 154.67 & $.016^{*}$ \\
\hline & Con $\times$ El & 28,560 & 2.15 & 0.71 & .100 \\
\hline \multirow[t]{2}{*}{ FC vs. IC } & Con & 1,20 & 0.43 & 9.09 & .521 \\
\hline & Con $\times \mathrm{El}$ & 28,560 & 2.30 & .65 & .080 \\
\hline \multicolumn{6}{|c|}{ Anterior vs. posterior $(2 \times 10$ electrodes $)$} \\
\hline FC vs. FI & Con $\times$ Site & 1,20 & 0.08 & 0.18 & .786 \\
\hline \multicolumn{6}{|c|}{ Anterior left vs. anterior right $(2 \times 5$ electrodes $)$} \\
\hline FC vs. FI & Con $\times$ Site & 1,20 & 0.05 & 0.05 & .820 \\
\hline \multicolumn{6}{|c|}{ Posterior left vs. posterior right ( $2 \times 5$ electrodes $)$} \\
\hline FC vs. FI & Con $\times$ Site & 1,20 & 0.44 & 0.45 & .515 \\
\hline \multicolumn{6}{|c|}{$\begin{array}{l}\text { Con }=\text { congruity type; } \mathrm{El}=\text { electrode. } \\
* p<.05 .\end{array}$} \\
\hline \multicolumn{3}{|c|}{972 Journal of Cognitive Neuroscience } & & \multicolumn{2}{|c|}{ Volume 13, Number 7} \\
\hline
\end{tabular}


Table 2. ANOVA on Mean ERP Amplitude in the 300-500-msec Latency Range (N400)

\begin{tabular}{|c|c|c|c|c|c|}
\hline & Source & $d f$ & $F$ & MSE & $p$ \\
\hline \multicolumn{6}{|c|}{ Omnibus ANOVA (29 electrodes) } \\
\hline \multirow[t]{2}{*}{ Overall } & Con & 2,40 & 47.71 & 1734.09 & $.000 * * *$ \\
\hline & Con $\times \mathrm{El}$ & 56,1120 & 8.96 & 4.34 & $.000 * * *$ \\
\hline \multirow[t]{2}{*}{ FC vs. FI } & Con & 1,20 & 66.46 & 3432.98 & $.000 * * *$ \\
\hline & Con $\times \mathrm{El}$ & 28,560 & 12.00 & 7.85 & $.000 * * *$ \\
\hline \multirow[t]{2}{*}{ FC vs. IC } & Con & 1,20 & 45.52 & 1185.66 & $.000 * * *$ \\
\hline & Con $\times \mathrm{El}$ & 28,560 & 9.22 & 4.19 & $.000 * * *$ \\
\hline \multirow[t]{2}{*}{ IC vs. FI } & Con & 1,20 & 18.62 & 583.62 & $.000 * * *$ \\
\hline & Con $\times \mathrm{El}$ & 28,560 & 2.82 & 0.97 & $.047^{*}$ \\
\hline \multicolumn{6}{|c|}{ Anterior vs. posterior $(2 \times 10$ electrodes $)$} \\
\hline FC vs. FI & Con $\times$ Site & 1,20 & 8.34 & 47.94 & $.009 * *$ \\
\hline FC vs. IC & Con $\times$ Site & 1,20 & 7.14 & 27.12 & $.015^{*}$ \\
\hline IC vs. FI & Con $\times$ Site & 1,20 & 0.95 & 2.95 & .340 \\
\hline \multicolumn{6}{|c|}{ Anterior left vs. anterior right $(2 \times 5$ electrodes $)$} \\
\hline FC vs. FI & Con $\times$ Site & 1,20 & 0.47 & 0.49 & .502 \\
\hline FC vs. IC & Con $\times$ Site & 1,20 & 0.52 & 0.46 & .481 \\
\hline IC vs. FI & Con $\times$ Site & 1,20 & 3.47 & 1.90 & .077 \\
\hline \multicolumn{6}{|c|}{ Posterior left vs. posterior right ( $2 \times 5$ electrodes $)$} \\
\hline FC vs. FI & Con $\times$ Site & 1,20 & 0.16 & 0.28 & .691 \\
\hline FC vs. IC & Con $\times$ Site & 1,20 & 0.81 & 1.03 & .379 \\
\hline IC vs. FI & Con $\times$ Site & 1,20 & 4.51 & 2.38 & $.046 *$ \\
\hline
\end{tabular}

baseline condition were significantly larger over posterior than anterior regions of the scalp. Only in the posterior left versus posterior right analysis was a hemispheric difference found between the IC and FI conditions, indicating that the difference in N400 amplitude is larger over right than left posterior areas.

To establish whether the unexpected difference in ERP amplitude between the FI and IC conditions might be due to an early differential effect in the N200 latency window, we performed additional analyses in the N400 latency window after averaging the waveforms in the 250-300-msec interval. This time interval corresponds to the period involving the offset of the N200 component. Figure 3 shows the grand average waveforms after this alternative normalization procedure. The omnibus ANOVA indicated that the basic congruity effect in the N400 latency window was still robust, $F(2,40)=16.70, M S E=$
458.19, $p<.001$. The pairwise comparisons revealed that both the FI and the IC completions elicited a larger N400 than the FC completions (both $p<.001$ ). However, the FC and the IC conditions did not significantly differ from each other, $F(1,20)<1$.

\section{The Late Positivity Latency Window: 600-1000 msec}

Table 3 displays the results of the mean ERP amplitude omnibus ANOVAs in the 600-1000-msec latency range. The omnibus ANOVA on mean amplitudes resulted in a significant main effect of congruity. Pairwise analyses revealed that the FC completions differed significantly from both the FI completions and the IC completions. The ERP amplitude in the FI condition also differed significantly from the ERP amplitude in the IC condition. 


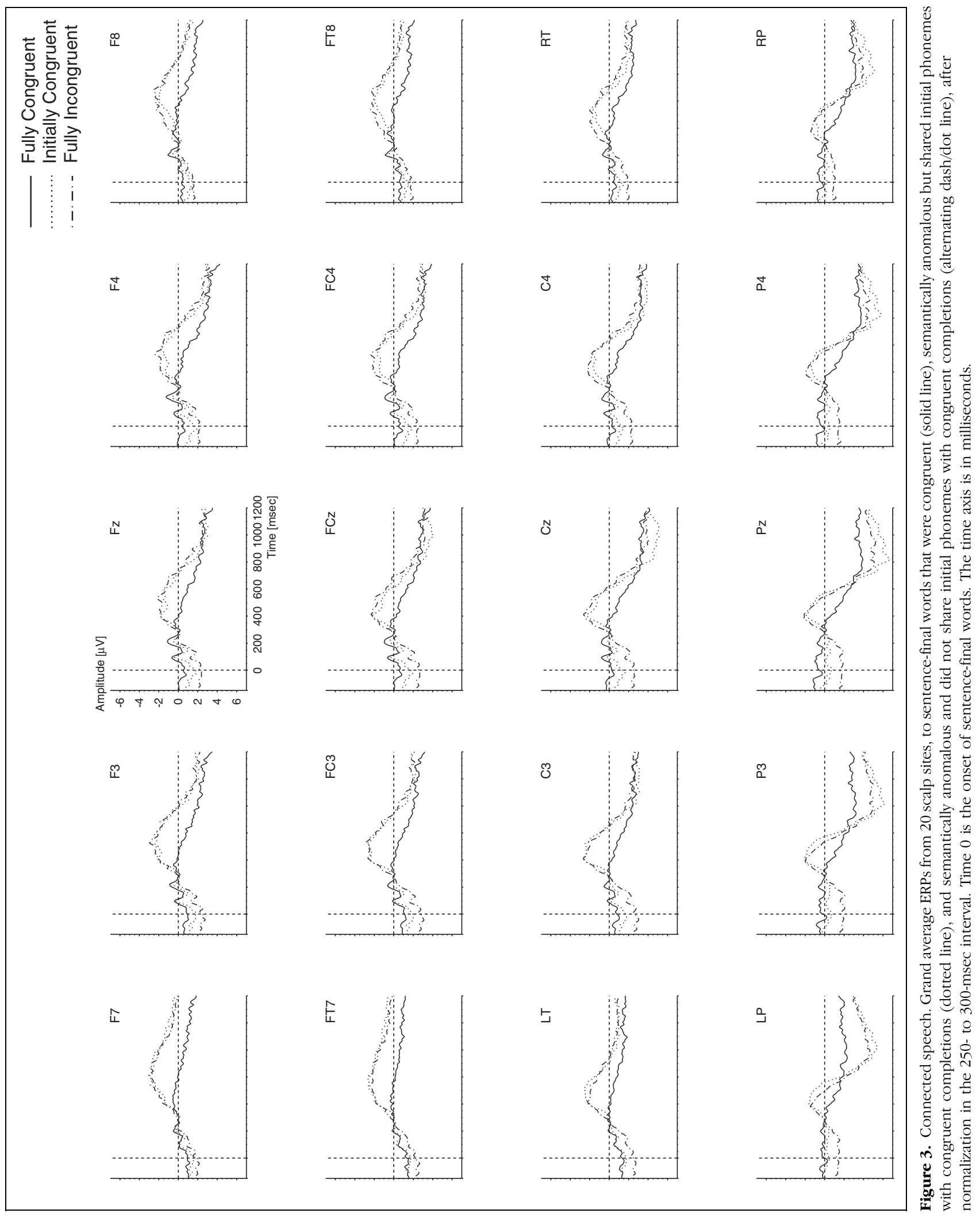


Table 3. ANOVA on Mean ERP Amplitude in the 600-1000-msec Latency Range

\begin{tabular}{|c|c|c|c|c|c|}
\hline & Source & $d f$ & $F$ & $M S E$ & $p$ \\
\hline \multicolumn{6}{|c|}{ Omnibus ANOVA (29 electrodes) } \\
\hline \multirow[t]{2}{*}{ Overall } & Con & 2,40 & 26.45 & 836.35 & $.000 * * *$ \\
\hline & Con $\times \mathrm{El}$ & 56,1120 & 26.83 & 17.24 & $.000 * * *$ \\
\hline \multirow[t]{2}{*}{ FC vs. FI } & Con & 1,20 & 36.44 & 1633.24 & $.000 * * *$ \\
\hline & Con $\times \mathrm{El}$ & 28,560 & 33.84 & 21.06 & $.000 * * *$ \\
\hline \multirow[t]{2}{*}{ FC vs. IC } & Con & 1,20 & 7.10 & 218.07 & $.015^{*}$ \\
\hline & Con $\times$ El & 28,560 & 32.47 & 28.12 & $.000 * * *$ \\
\hline \multirow[t]{2}{*}{ IC vs. FI } & Con & 1,20 & 33.99 & 657.73 & $.000 * * *$ \\
\hline & Con $\times \mathrm{El}$ & 28,560 & 5.80 & 2.55 & $.001^{* * *}$ \\
\hline
\end{tabular}

Again, because of the possibility of amplitude effects in this latency window as a consequence of earlier differential effects in the N200 latency window, we performed further analyses after the alternative normalization procedure in the 250-300-msec interval.

Table 4 displays the results of the mean ERP amplitude omnibus ANOVAs in the 600-1000-msec latency range after having normalized the waveforms in the 250300-msec interval (Figure 3). The omnibus ANOVA on mean amplitudes did not result in a significant main effect of congruity. None of the conditions differed significantly from each other. However, Figure 3 reveals that this is possibly due to the difference in effects between the anterior and posterior electrodes. Topographical analyses indeed revealed that the effects in relation to the $\mathrm{FC}$ condition (baseline) had a parietal distribution, and were moreover largest over the left hemisphere: In the anterior/posterior and posterior left/ posterior right analyses, the interaction of the factors congruity by site reached significance both in the FC versus FI comparison and the FC versus IC comparison.

\section{Onset Latencies}

Figure 1 shows that the onset of the effects is most clearly visible over frontal electrodes. Therefore, we decided to analyze this frontal band. Onset latencies were estimated by first separately computing the mean amplitude values for five frontal electrodes (Fz, F4, F3, F8, and F7) in 20msec latency ranges (bins) that shifted in steps of 10 msec from target onset until 500 msec after target onset (e.g., 0-20, 10-30, etc.). The values for the latency bins were submitted to ANOVAs that tested against the null hypothesis of zero difference between the a priori selected conditions FC versus FI and FC versus IC.
The onset latency analyses for the FC versus FI comparison on the amplitudes of the two waveforms in the three consecutive bins of 140-160, 150-170, and 160-180 msec revealed a significant congruity effect $(p=.05, p=.02, p=.02$, respectively). This indication of an early congruity effect disappeared in the following bins but emerged again at $220 \mathrm{msec}$, at which point the congruity effect remained significant over the entire test region ( $500 \mathrm{msec}$ ). In the $\mathrm{FC}$ versus $\mathrm{IC}$ comparison, the congruity effect did not start until $270 \mathrm{msec}$. So, at 150180 msec after word onset, the waveforms of the FI condition momentarily departed from the FC condition. After this short-lived congruity effect, the waveforms of the FI condition significantly increased in amplitude approximately $50 \mathrm{msec}$ earlier than the waveform of the IC words (i.e., 220 vs. $270 \mathrm{msec}$ after word onset).

\section{N200 versus $\mathbf{N} 400$}

Figure 4 shows the topography of mean amplitude effects between the FC and FI conditions in the N200 and the N400 window after our standard normalization procedure (150 msec prestimulus interval). Although the N200 component is most clearly visible over frontal sites, the figure reveals that the N200 effect is equally distributed across the scalp, whereas the N400 effect is largest over centro-parietal sites. To establish whether these two congruity effects have statistically distinct scalp distributions, we performed topographical analyses. First, for every subject, difference scores between the FC and FI conditions were computed for every electrode in the N200 latency window and the N400 latency window. Second, a scaling procedure was performed to avoid that differential amplitude effects between the two latency windows would be incorrectly 
Table 4. ANOVA on Mean ERP Amplitude in the 600-1000-msec Latency Range after Averaging in the 250-300-msec Interval

\begin{tabular}{|c|c|c|c|c|c|}
\hline & Source & $d f$ & $F$ & MSE & $p$ \\
\hline \multicolumn{6}{|c|}{ Omnibus ANOVA (29 electrodes) } \\
\hline \multirow[t]{2}{*}{ Overall } & Con & 2,40 & 1.26 & 55.92 & .292 \\
\hline & Con $\times$ El & 56,1120 & 22.84 & 15.36 & $.000 * * *$ \\
\hline \multirow[t]{2}{*}{ FC vs. FI } & Con & 1,20 & 1.73 & 107.26 & .204 \\
\hline & Con $\times \mathrm{El}$ & 28,560 & 28.49 & 20.91 & $.000 * * *$ \\
\hline \multirow[t]{2}{*}{ FC vs. IC } & Con & 1,20 & 0.33 & 11.05 & .569 \\
\hline & Con $\times \mathrm{El}$ & 28,560 & 23.18 & 79.97 & $.000 * * *$ \\
\hline \multirow[t]{2}{*}{ IC vs. FI } & Con & 1,20 & 1.30 & 49.46 & .268 \\
\hline & Con $\times \mathrm{El}$ & 28,560 & 1.77 & 0.72 & .142 \\
\hline \multicolumn{6}{|c|}{ Anterior vs. posterior $(2 \times 10$ electrodes $)$} \\
\hline FC vs. FI & Con $\times$ Site & 1,20 & 53.16 & 269.51 & $.000 * * *$ \\
\hline FC vs. IC & Con $\times$ Site & 1,20 & 68.67 & 360.33 & $.000 * * *$ \\
\hline IC vs. FI & Con $\times$ Site & 1,20 & 2.89 & 6.58 & .104 \\
\hline \multicolumn{6}{|c|}{ Anterior left vs. anterior right $(2 \times 5$ electrodes $)$} \\
\hline FC vs. FI & Con $\times$ Site & 1,20 & 0.01 & 0.01 & .938 \\
\hline FC vs. IC & Con $\times$ Site & 1,20 & 1.24 & 2.24 & .279 \\
\hline IC vs. FI & Con $\times$ Site & 1,20 & 3.04 & 1.97 & .097 \\
\hline \multicolumn{6}{|c|}{ Posterior left vs. posterior right $(2 \times 5$ electrodes $)$} \\
\hline FC vs. FI & Con $\times$ Site & 1,20 & 23.49 & 38.40 & $.000 * * *$ \\
\hline FC vs. IC & Con $\times$ Site & 1,20 & 12.74 & 26.15 & $.002 * *$ \\
\hline IC vs. FI & Con $\times$ Site & 1,20 & 1.39 & 1.17 & .252 \\
\hline
\end{tabular}

interpreted as distribution effects. In this procedure, the electrode-specific difference scores were $z$-transformed for each latency window separately (Rösler, Heil, \& Glowolla, 1993; see also McCarthy \& Wood, 1985). The $z$-transformed values were entered into an ANOVA that crossed the 2-level latency window factor (N200 and N400) with the 29-level electrode factor. A significant interaction of latency window by electrodes, $F(28,560)=6.01, M S E=2.19, p<.001$, revealed that the congruity effects found in the two latency windows indeed have different spatial distributions across the scalp. This difference in scalp distributions was subsequently explored in three additional topographical ANOVAs (using the same electrode configurations as previously described). In the anterior/posterior analysis, a significant interaction of window by site was obtained, $F(1,20)=9.61, M S E=20.84, p<.01$. In the anterior left/right and the posterior left/right analyses, no significant interaction of window by site was obtained (both $F$ S $<1$ ). The significant interaction of window by site in the anterior/posterior analysis underscores that the N200 and N400 effects have different spatial distributions, with the N400 effect having a more posterior distribution and the N200 effect having a flat distribution across the scalp.

\section{Time-Locking to the Divergence Point}

Figure 5 displays the grand average waveforms by electrode site time-locked on a trial-by-trial basis to the time point at which the sentence-final words in the FC and IC conditions started to acoustically diverge from each other. This acoustical divergence point was assessed on the basis of phonetic transcriptions of the sen- 
Figure 4. Connected speech. Distribution of the N200 and N400 effects for four left hemisphere sites (F3, FC3, C3, P3), four midline sites (Fz, $\mathrm{FCz}, \mathrm{Cz}$, $\mathrm{Pz}$ ), and four right hemisphere sites (F4, FC4, C4, P4). The N200 effect was determined by subtracting the mean amplitude in the 150-250-msec latency window of the grand average ERP for the semantically congruent sentence-final words from the mean amplitudes of the grand average ERP for the semantically anomalous sentence-final words that did not share the same initial phonemes as the semantically congruent words. The N400 effect was determined in the same manner on the basis of the mean amplitudes in the 300- to 500 -msec latency window.

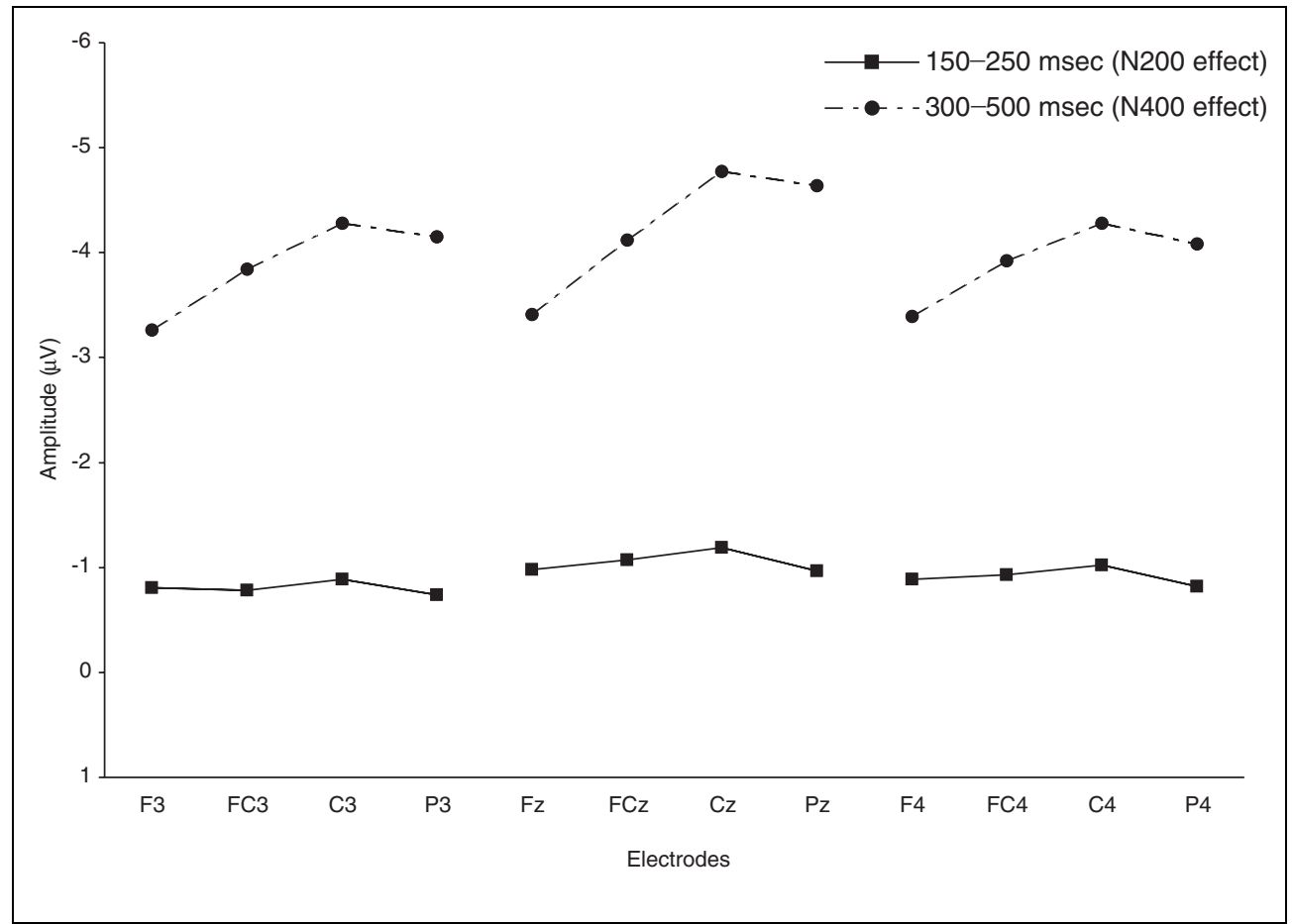

tence-final words. The time period preceding this divergence point corresponds to the mean time period of segmental overlap between the two conditions, which was $220 \mathrm{msec}$. Therefore, in the alignment in Figure 5, the zero point roughly corresponds to word onset. However, since information about the exact onset of each sentence-final word is lost in this time-locking procedure, normalization cannot take place on the basis of information related to word onset. Therefore, the waveforms were normalized on the basis of the averaged activity of $150 \mathrm{msec}$ preceding the divergence point. What can be seen in this figure is that approximately 80 msec after the divergence point, the waveform of the IC condition shows a steep ascending flank, resulting in what appears to be a biphasic N400. ${ }^{3}$ This biphasic morphology is most apparent at the frontal and frontocentral electrodes. Parietal electrodes reveal that the N400 component in the IC condition is followed by a late positive shift.

\section{DISCUSSION}

This study investigated the time course of contextual influences on spoken-word recognition. We used ERPs to investigate the presence of markers in the electrophysiological signal that reveal the moment at which context starts to have an effect on word recognition. We hypothesized that if context exerts an influence early on in the signal, the ERP of the FI condition should diverge earlier than the ERP of the IC condition, compared to the FC condition. The moment at which the FI condition starts to diverge provides an upper estimate of the onset of contextual influences on spoken-word recognition. As hypothesized, the onset of the congruity effect between the FC and the FI conditions preceded that of the FC and the IC conditions. The waveforms of the FI condition first diverged momentarily from the FC condition in the latency interval of 140-180 msec. This short-lived effect disappeared, but emerged again at $220 \mathrm{msec}$ and persisted throughout the entire test region. The congruity effect between the FC and IC conditions did not start until $270 \mathrm{msec}$. These results indicate that at least at $220 \mathrm{msec}$, but possibly already at $140 \mathrm{msec}$, sentential context has an influence on the auditory word recognition process.

We were particularly interested in the manifestation of this congruity effect. Would it be a monophasic N400 effect as in the Van Petten et al. study (1999), or a biphasic negative shift consisting of an early negative shift and an N400 effect as in the Connolly and Phillips study (1994) and the Hagoort and Brown study (2000)? These ERP profiles can be linked to different functional interpretations of the elicited components. The single N400 effect hypothesis suggests that the monophasic N400 effect is a reflection of an overall semantic processing, without fractionation into lexical selection and integration. The alternative position suggests that the early negative shift and the N400 effect are two distinct effects, reflecting different aspects of the spoken-word recognition process. Our grand average waveforms (Figures 1 and 2) revealed two clear negative deflections, one peaking at $200 \mathrm{msec}$ and the other at $400 \mathrm{msec}$. This supports the hypothesis of a separate effect preceding the N400 in the auditory domain. 


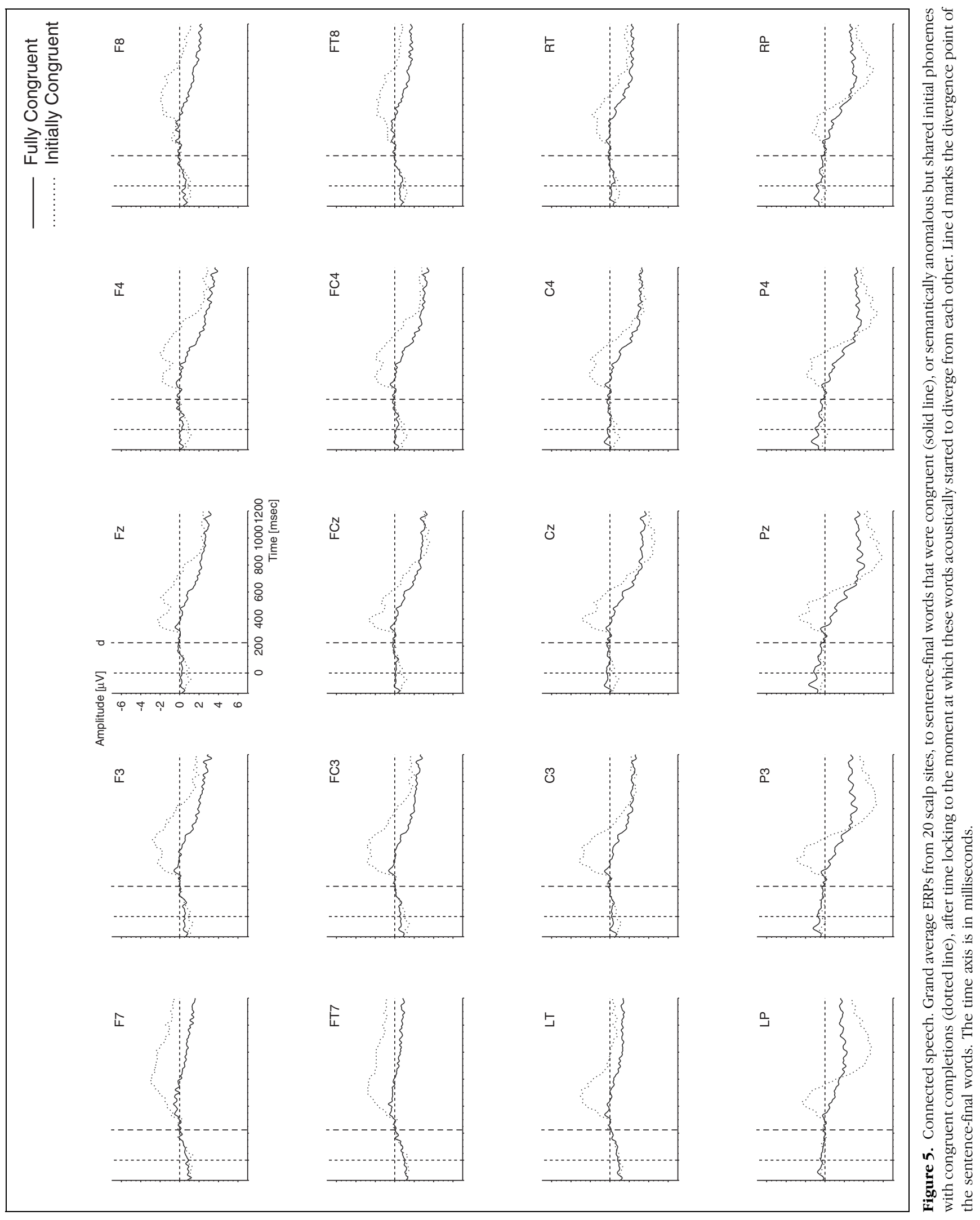


The early negativity or N200 component was visible in all three conditions with its morphology most pronounced over frontal sites. Statistical analyses revealed that the N200 in the FI condition was larger than the N200 in the FC condition and that the latter did not differ in amplitude from the N200 obtained in the IC condition. The finding that an early negativity could be obtained in all three conditions with the amplitude differentially modulated by the experimental conditions could be an indication that the N200 effect reflects a distinct aspect of the spoken language comprehension process.

However, the single N400 effect hypothesis could still be maintained on the basis of the obtained electrophysiological profile. One could argue that the N200 effect is not a separate effect, but instead is the first part of a biphasic N400 effect, and that this biphasic N400 effect reflects an undifferentiated semantic process. This would imply that the modulation brought about by the experimental conditions is due to a differential onset of the biphasic N400. We report several findings that provide evidence for the claim that the early negative shift is in fact a separate effect.

The notion that the N200 effect is a separate effect was enhanced by the dissociation with the N400 effect in the IC condition relative to the FC condition. The N200 did not differ in amplitude; however, we did obtain a highly significant N400 effect, with a large N400 amplitude in the IC condition compared to the FC condition. This finding by itself could also be accounted for by the biphasic N400 effect hypothesis. One could argue that the difference in onset of the congruity effects is due to the N400 component setting in later, namely when the anomaly is first detected. However, if the early negativity is an integral part of the electrophysiological profile of the N400 that varies in onset, then the entire biphasic negative deflection should shift in time as a function of anomaly detection. In other words, in the IC condition, where the anomaly is detected later compared to the FI condition, the early negativity should also occur later. This is not the case.

The alternative time-locking procedure that aligned the waveforms of the FC and IC conditions to the moment at which these two signals started to acoustically diverge (the divergence point) provides information about the morphology of the onset of the congruity effects. Figure 5 revealed that at the frontal sites, where the morphology of the N200 was most pronounced, the N400 in the IC condition had a steep and uniform ascending slope. This is in contrast to the manifestation of the congruity effect in the FI condition in Figure 1, where an early negativity was obtained in the ascending slope. The finding that after the alternative time-locking procedure the N400 in the IC condition was not preceded by a component similar to the early negativity in the FI condition indicates that the early negative shift reflects a process that occurs at or around $200 \mathrm{msec}$ after word onset and is not part of the N400 effect.
Finally, the topographical anterior versus posterior analysis after scaling of the absolute size of the effects revealed that the $\mathrm{N} 200$ and N400 effects have significantly different spatial distributions. Figure 4 shows that the N200 effect had an equal distribution from front to back, whereas the N400 effect had a centro-parietal distribution. Statistically significant differences in scalp distribution are usually interpreted as reflecting the activity of at least partly distinct neuronal populations.

Taken together, these four findings, (a) an early negativity visible in all conditions, but larger in the FI condition, (b) the absence of an early negativity effect, together with the presence of an N400 effect in the IC condition relative to the FC condition, (c) the early negativity strictly time-locked to word onset and not affected by the divergence point, and (d) the different scalp distributions of the early negative shift and the N400 effect, provide evidence in support of the hypothesis that the N200 effect is separate from and precedes the N400 effect.

The presence of an early negativity is in line with the findings of Connolly and Phillips (1994) and Hagoort and Brown (2000). However, it does not provide evidence for the account given by Connolly and Phillips. They assumed that on the basis of the sentence context an expectation of a particular word would be formed, and that the early negativity would be elicited when the initial phonemes of the perceived word did not match the initial phonemes of the expected word. The major finding that argues against this account is the elicitation of an N200 in all of our conditions, irrespective of whether the phonemes corresponded to the initial phonemes of the highest cloze word. Therefore, the N200 component cannot truly be a PMN. Even though we cannot rule out the possibility that contextual information can be used on-line to specifically predict a unique lexical item, the conclusion by Van Petten et al. (1999) seems more compatible with our findings. They concluded, on the basis of an onset of the N400 that could precede the eliciting word's IP, that semantic processing begins on partial and incomplete information about the perceived word. However, instead of an early onset of the N400 effect, a separate negative shift preceding the $\mathrm{N} 400$ effect was obtained in our study as well as in the recent study by Hagoort and Brown (2000). It is not clear why our findings differ in this respect from those of Van Petten et al., especially since the two studies are rather similar. An important difference between the studies could be that all of our sentence-final words began with plosives, whereas Van Petten et al. used many different consonants. The use of plosives in our study makes the onsets of the sentencefinal words more similar. The clear N100 component in our waveforms provides evidence for this. Although we used natural connected speech, after averaging across trials, the waveforms still revealed a clear exogenous marker of word onset. Similarly, the chances of finding 
the separate N200 effect may become greater if word onsets across trials are fairly homogeneous. In most other studies, different consonant types have been used, which might have introduced a latency jitter in the averaged waveforms, masking potential N200 effects.

The early negativity we obtained is reminiscent of another negativity that emerges in the 200-msec range, the mismatch negativity (MMN). This component is related to physical mismatches in primary auditory processing (cf. Näätänen, 1990; Näätänen \& Alho, 1997). However, besides the primary fact that the manipulation in our experimental conditions was semantic in nature rather than physical, there are several other arguments why our obtained N200 effect is not an MMN. First, the MMN has a topographical distribution that is different from the observed N200 effect; it is maximal at frontal electrode sites, whereas the N200 effect has a flat distribution across the scalp. Second, it shows a polarity inversion at the lateral electrodes, which is absent in the N200 in our study. Third, the electrophysiological profile of the MMN is different. It has an earlier onset and is not as sharply peaked as the N200. Therefore, we propose that the early negative shift in our study has a functionality that is distinct from both the MMN and the N400 effect and that reflects a process in word recognition that precedes the integration of a selected word into the sentential context. ${ }^{4}$

We envisage the spoken-word recognition process as follows (cf. Norris, 1994; Zwitserlood, 1989; MarslenWilson \& Welsh, 1978): On the basis of an analysis of the initial phoneme(s) of the spoken word a number of lexical candidates are accessed. This is a purely formdriven, bottom-up process. After activation of these candidates, top-down context information starts to exert its influence. On the basis of their semantic and syntactic features, candidates in the set are assessed with respect to their goodness-of-fit within the sentence frame. In the presence of semantic features in the activated set that fit the sentence context well, further incoming acoustic information and top-down contextual information are used to narrow down the number of candidates to the one that is most compatible with both form and content constraints. This candidate is subsequently integrated in the sentence context. If, however, none of the lexical candidates fit the context well and consequently the appropriate semantic features are not available, selection of the proper candidate is difficult and can only be done on the basis of the acoustic information. Once the incongruent word is selected from the set of candidates, integration is attempted. In this sense, semantic integration is a mandatory process: Integration will be attempted for all the words in a sentence.

It has been widely assumed that the amplitude of the N400 indicates whether the integration process runs smoothly: A small N400 indicates that integration of the selected word is easy, a large N400 indicates that integration is difficult. This account is supported by our results: We obtained a small $\mathrm{N} 400$ in the FC condition and a significantly larger N400 in the two semantically incongruent conditions. We propose that the amplitude modulation of the early negativity preceding the N400 effect reflects the lexical selection process that occurs at the interface of lexical form and contextual meaning (cf. Hagoort \& Brown, 2000). Analogous to the functional interpretation of the N400, the amplitude of the N200 is indicative of whether the initial assessment of the formbased activated lexical candidates reveals the presence of the semantic features that are required by the contextual specifications. A small N200 is elicited when the set contains semantic features that fit the sentence context (as in FC and IC), a large N200 indicates that the set does not contain semantic features that fit the preceding sentence frame well (as in FI).

The exact nature of this early assessment process is not known. After an analysis of the initial sounds of a word, a number of lexical candidates are activated. Upon activation of these words, many semantic features that are associated with them become instantly available to the semantic processing system. At present it is impossible to distinguish between the following two accounts: Either the lexical candidates are individually assessed with respect to their goodness-of-fit within the sentence frame on the basis of their semantic features (a lexicaldriven process) or initial assessment takes place on the basis of a field of semantic features that at that moment in time are not linked to specific lexical candidates in the set (feature-driven). The former account assumes that an increase of the N200 amplitude is seen when none of the lexical candidates fit the sentential context, whereas the latter assumes that a larger N200 is elicited when the semantic features that are required for congruity in the sentence frame are not (all) among the field of activated semantic features.

Although these two functional accounts of the N200 effect are speculative at this point, it is important to note that they do make different and testable predictions about the processing of words that vary in their contextual constraint in sentence contexts. The results of the present study show that in relatively highly constraining sentences, congruent words elicit both a small N200 and a very reduced N400. In contrast, incongruent words elicit a larger N200 and an increased N400. There is evidence in the literature that the N400 is sensitive to contextual constraint. For example, taking into account that in semantically congruent sentences contextual constraint and cloze probability are tightly linked phenomena (cf. Kutas, Lindamood, \& Hillyard, 1984), the studies by Kutas and Hillyard (1984) and Van Petten et al. (1999) reveal a sensitivity of the N400 to variations of contextual constraint. In these studies, when the contextual constraint of a sentence was low, congruent words (by definition of low cloze probability) elicited an N400 that was larger 
in amplitude than the N400 elicited to (high cloze) congruent words in highly constraining sentences. This indicates that although a word is congruent, the absence of highly constraining context makes the integration process to some extent more demanding. Suppose that, in accordance with the lexical-driven account of the $\mathrm{N} 200$, individual lexical candidates are assessed with respect to their possible fit within the preceding sentence. Analogous to the N400, we would expect a relatively large N200 to occur in response to congruent words in low-constraint sentences: The absence of strong contextual constraints makes it more difficult to assess whether any candidate in the activated set fits the sentence frame very well. Note that this account also predicts a larger N200 in response to congruent words at earlier positions in the (high- or low-constraint) sentence, since contextual constraint is absent at the beginning of the sentence and increases as the sentence unfolds. However, if the modulation of the N200 amplitude reflects a process in which the presence of appropriate semantic features is assessed, we would expect a small N200 to occur, because most semantic features are compatible with low constraining sentences.

The functional interpretation of the N200 effect as reflecting an initial assessment process (irrespective of the feature- or lexical-driven account) also makes some predictions about words differing in cloze probability in equally constraining sentences. The present study revealed that high-cloze probability words elicit an N200 that is small in amplitude. From our claim, it follows that words of a lower cloze probability should elicit larger N200s, since the lexical candidates or the field of semantic features in the activated set would not be considered to fit the sentence context very well. Words with low cloze probabilities (in low-constraint sentences) should produce the largest N200, possibly very close to the N200 elicited by fully anomalous words in high-constraint sentences. These predictions need to be evaluated in future research.

An issue that we have not yet touched upon but needs some clarification is the apparent differential N400 effect between the FI and IC conditions. It is not immediately clear why two sentence-final words that are both anomalous in a sentence frame should elicit N400s that differ in amplitude. One possibility is that the N400 effect is due to a lexical frequency effect, since the mean frequency of the words used in the two conditions differed, albeit only slightly (a log frequency of 2.62 per 42 million in IC and 2.49 per 42 million in FI). However, the work by Van Petten and Kutas (1990, see also Van Petten, 1995) has shown that frequency effects dissipate over the course of a sentence. Although low frequency words are usually associated with larger N400s than high frequency words, this frequency effect disappears when contextual constraint is increased as the sentence unfolds. Since the mean frequency of our critical words did not greatly differ across conditions and since these words were presented in sentence-final position, a lexical frequency effect does not seem to be a viable account for the N400 effect that we obtained.

Another suggestion comes from a word priming study by Praamstra, Meyer, and Levelt (1994). They examined phonological priming effects in an auditory lexical decision task and found that the ERP waveforms were more negative for unrelated targets than for alliterating targets (e.g., beeld-mast [statue-mast] vs. beeld-beest [statueanimal]). Also, in another experiment waveforms to unrelated targets were more negative than to rhyming targets (e.g., graaf-steeg [duke-alley] vs. graaf-staaf [duke-rod]). Praamstra et al. concluded that the N400 amplitude is sensitive to phonological as well as semantic manipulations. It is unclear how these form-priming effects for words presented in isolation relate to the processing of words in sentences. Note that in the study by Van Petten et al. (1999), the phonological manipulation in the rhyming condition did not elicit an N400 of lower amplitude than in the anomalous condition.

We propose a different view of the apparent N400 effect, namely that the observed shift is carried over from the preceding N200 effect. Figure 2 shows that the onset of the N400 in the FI and IC conditions occurs at about $275 \mathrm{msec}$. As a result of the N200 effect, at $275 \mathrm{msec}$ the FI condition is already larger in amplitude than in the IC condition. This differential N200 effect between the two conditions might then lead to an add-on amplitude effect in the N400 latency window. We performed additional analyses in this latency window after normalizing the waveforms in the 250- to 300-msec latency interval to remove the differential N200 effect between the FI and IC conditions, to see what the effect would be on the waveforms in the N400 latency window. Figure 3 shows that after normalization in the 250- to 300 -msec interval, the relative amplitudes of the two conditions actually did not differ from each other, and the statistical analyses revealed that an N400 effect was in fact absent.

Figure 3 also shows that after the alternative normalization procedure in the 250 - to 300 -msec interval, the difference in the size of the late positivity effect that we observed between the FI and IC conditions disappears. This was confirmed by our statistical analyses. Late positivity effects to sentence-final words are thought to be related to postlexical wrap-up processes (cf. Juottonen et al., 1996). Presumably, after the sentence-final word has been integrated in the context, an evaluation of the overall message takes place. The late positivity, which is largest in the FI and IC conditions, indicates that overall evaluation of the sentence is difficult, since sentence-final words in these conditions were anomalous.

Overall, the results obtained in this experiment suggest that the N200 effect reflects a process that occurs at 
the interface of lexical form and contextual meaning, in which form-based-activated lexical candidates are assessed with respect to their semantic goodness-of-fit with the sentential contextual specifications. After this early assessment process, selection of one specific candidate takes place on the basis of additional acoustic input and supporting context. The N400 effect reflects semantic integration of the selected candidate into the higher-order meaning of sentence context. Even though it remains possible that context already has an influence before any bottom-up information has been received, our findings seem most compatible with word identification models that claim that there is bottom-up priority of acoustic processing of the initial signal of the perceived word before top-down information exerts its influence.

\section{METHODS}

\section{Subjects}

The experiment was conducted with 21 native speakers of Dutch (18 female, mean age 22, range 19-26 years) from the subject pool of the Max Planck Institute for Psycholinguistics. All had normal to corrected-to-normal vision and were right-handed (three subjects reported having a left-handed parent or sibling). None of the subjects had any neurological impairment or had experienced any neurological trauma. Nor had any of the subjects participated in the pretest (see below). The data of five additional subjects were not analyzed (see Results section). Subjects were paid for their participation.

\section{Materials}

The experimental items consisted of a set of 261 triplets of semantically constraining sentences across three experimental conditions. The sentences in each triplet were identical up to the final word. In the FC condition, sentences ended with a high-cloze probability word, e.g., "De schilder kleurde de details in met een klein penseel" ("The painter colored the details with a small paint brush"). In the IC condition, sentences ended with a semantically anomalous word that had the same initial phonemes (and lexical stress) as the highest-cloze probability word, e.g., "De schilder kleurde de details in met een klein pensioen" ("The painter colored the details with a small pension"). The third condition served as a control condition where sentences ended with a word that was semantically anomalous and also had initial phonemes that were totally unexpected given the sentential context, e.g., "De schilder kleurde de details in met een klein doolbof" ("The painter colored the details with a small labyrinth"). This condition is called the FI condition.
The 261 triplets were derived from a larger set of sentence materials that had been submitted to a cloze test. Thirty subjects from the subject pool of the Max Planck Institute for Psycholinguistics were given a list of 350 sentences with the final word omitted. Subjects were asked to carefully read the sentences and to fill in the first word that came to mind at the position of the omitted sentence-final word. On the basis of the results, a set of 261 sentences with a cloze probability of .50 or higher formed the basis for the 261 triplets used in this experiment. Items for the IC condition were constructed by replacing the sentence-final word by a word that did not fit the semantic constraints of the preceding context, but began with the same phonemes (mean length of overlap was 2.6 phonemes, range $2-4$, always including a full vowel) and had the same lexical stress and number of syllables as the high-cloze probability word, which was used in the FC condition. The items for the FI condition were constructed by replacing the sentence-final word with a semantically anomalous word with word-initial phonemes that differed from the beginning phonemes of the high-cloze probability word and other congruent words.

Because the sentences within each triplet were identical up to the final word, they had the same length (mean length of 10.8 words; range 5-15) and were equally constraining; the critical words in the FC condition had a mean cloze probability of .84 (range: .501.0). All sentence-final words were reasonably wellknown nouns selected from a Dutch word corpus (CELEX, 1990); all critical words had a frequency of at least 30 counts per 42 million or $1.48 \mathrm{log}$, with a mean duration of $516 \mathrm{msec}$ and a mean log frequency of 2.87 in the FC condition, $538 \mathrm{msec}$ and 2.62 in the IC condition, and $515 \mathrm{msec}$ and 2.49 in the FI condition. Each sentence-final word began with a plosive. This provided a clear physical marker that facilitated the alignment of the ERP waveform to the onset of the sentence-final word.

Three lists were constructed to ensure that no subject heard the same sentence or target word more than once. In addition to the set of 261 experimental items (87 per condition per list), a set of 87 fillers was constructed. These fillers were all semantically congruous, to balance the number of sentences that ended anomalously and congruously. Finally, the experimental lists were preceded by a practice list of 20 items, which reflected the experimental materials. The practice list was used to familiarize the subjects with the experimental situation.

The experimental sentences, fillers, and practice sentences were all spoken by a female speaker, with normal intonation at a normal speaking rate, and were recorded in one recording session. The sentences were recorded in triplets, making sure intonation and speaking rate were kept constant within each triplet. No specific voice changes marked the sentence-final anomalies as odd in 
the IC and FI conditions. Sentences were spoken in a sound-attenuating booth and recorded on a digital audiotape. The DAT recordings were sampled at 16$\mathrm{kHz}$ mono and stored on computer disk. A speech waveform editing system was used to mark the onset of the sentence-final words. Also, the moment at which the sentence-final words in the FC and IC conditions started to acoustically diverge from each other was marked as the divergence point for each word individually. This acoustic divergence point was assessed on the basis of phonetic transcriptions of the sentence-final words.

\section{Procedure}

Subjects were tested individually in a dimly illuminated sound-attenuating booth. They were seated in a comfortable reclining chair, instructed to move as little as possible, and told that they would hear a number of sentences. Their task was to attentively listen to these sentences and to try to understand them. In addition, they were told that several sentences would be semantically anomalous. No additional task demands were imposed.

Each trial began with a 300-msec warning tone, followed by $1200 \mathrm{msec}$ of silence, then a spoken sentence. The next trial began $4100 \mathrm{msec}$ after the sentence offset. To ensure that subjects would not blink during and shortly after presentation of the sentence, $1000 \mathrm{msec}$ prior to the beginning of the sentence, a fixation point (an asterisk) was displayed on the computer screen. The asterisk remained on the screen until 1600 msec after offset of the spoken sentence. Subjects were instructed to fixate on the asterisk during presentation of the sentences, but were free to blink and move their eyes when the asterisk was not displayed on the screen. Subjects listened to the stimuli via closed-ear headphones. After the practice session, the trials were presented in five blocks of approximately $10 \mathrm{~min}$.

\section{EEG Recording}

The EEG was recorded from $29 \mathrm{Ag} / \mathrm{AgCl}$-sintered electrodes mounted in an elastic cap, each referred to the left mastoid (see Figure 6 for the electrode distribution). Five electrodes were placed according to the 10\% standard system of the American Electroencephalographic Society over midline sites at Fz, $\mathrm{FCz}, \mathrm{Cz}, \mathrm{Pz}$, and $\mathrm{Oz}$ locations, along with nine lateral pairs of electrodes over standard sites on frontal (AF3, AF4, F3, F4, F7, and F8), fronto-central (FC3 and FC4), fronto-temporal (FT7 and FT8), central (C3 and $\mathrm{C} 4$ ), centro-parietal (CP3 and $\mathrm{CP} 4)$, parietal (P3 and $\mathrm{P} 4$ ), and occipital (PO7 and PO8) positions. Three additional pairs were placed laterally over symmetrical positions: (a) a temporal pair (LT and RT)

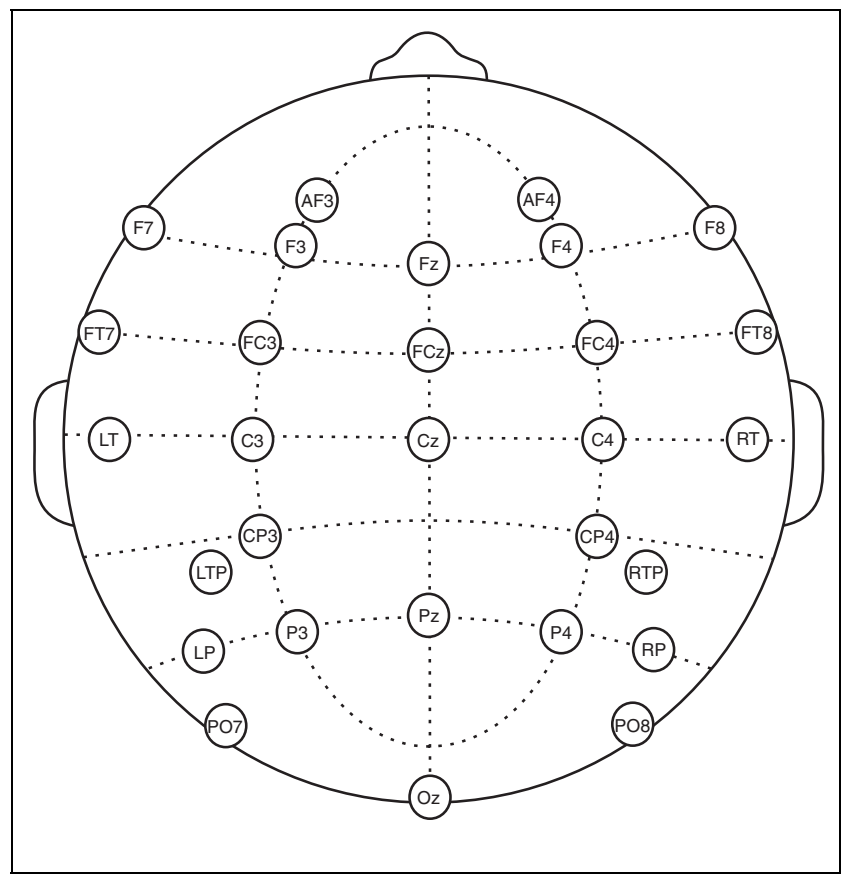

Figure 6. Distribution of the 29 electrodes across the scalp.

placed laterally to $\mathrm{Cz}$, at $33 \%$ of the interaural distance, (b) a temporo-parietal pair (LTP and RTP) placed $30 \%$ of the interaural distance lateral and 13\% of the nasion-inion distance posterior to $\mathrm{Cz}$, and (c) a parietal pair midway between LTP/RTP and PO7/PO8 (LP and RP). Vertical eye movements were monitored via a supra- to suborbital bipolar montage. A right to left canthal bipolar montage was used to monitor for horizontal eye movements. Activity over the right mastoid bone was recorded on an additional channel to determine if there were differential contributions of the experimental variables to the presumably neutral mastoid site. No such differential effects were observed.

The EEG and EOG recordings were amplified with a SynAmp Model 5083 EEG amplifier (NeuroScan, Herndon, VA, USA), using a hi-cut of $30 \mathrm{~Hz}$ (notch filter 60 $\mathrm{Hz})$ and a time constant of $8 \mathrm{sec}(0.02 \mathrm{~Hz})$. Electrode impedances were kept below $3 \mathrm{k} \Omega$ for the EEG recording and below $5 \mathrm{k} \Omega$ for the EOG recording. The EEG and EOG signals were digitized on-line with a sampling frequency of $200 \mathrm{~Hz}$.

\section{Acknowledgments}

We thank Jos van Berkum and Michael Coles for their support, Valesca Kooijman and Jelle van Dijk for their help in acquiring the ERP data, Marlies Wassenaar for recording the sentences, René de Bruin, John Nagengast, and Johan Weustink for technical assistance, and three anonymous reviewers for comments on an earlier version of the manuscript.

Reprint requests should be sent to Daniëlle van den Brink, Max Planck Institute for Psycholinguistics, P.O. Box 310, NL-6500 AH Nijmegen, The Netherlands, or via e-mail: danvdb@mpi.nl. 


\section{Notes}

1. Cloze probability of a word is defined as the percentage of subjects who use that word to end a particular sentence (see Bloom \& Fischler, 1980).

2. We do not expect a differential N100 effect to occur (sentences and task demands were identical in all conditions), and in fact this amplitude difference did not reach significance $(p=.103)$. Nevertheless, we were concerned that the IC condition in later latency windows might still be affected by this early difference. To assess the possible effect of the nonsignificant N100 amplitude difference, we normalized with a latency window surrounding the N100, and performed full statistical analyses based on this alternative normalization procedure. Apart from a few details in the onset analyses (IC now diverged at $370 \mathrm{msec}$ from FC), the results on the basis of this alternative normalization procedure were the same as the results based on the prestimulus baseline.

3. Investigation of this biphasic $\mathrm{N} 400$ revealed that the second peak is related to word offset. When we time-locked the waveforms to word offset, we discovered a large negative peak at word offset, followed by a smaller positive peak in the signal. This profile was apparent in all three conditions. This leads us to posit that we are not looking at a biphasic N400, but rather a monophasic N400 followed by an offset potential on the descending flank of the $\mathrm{N} 400$.

4. We do not claim that the N200 effect is language-specific (cf. Pritchard, Shappell, \& Brandt, 1991), but rather that in the context of spoken-word processing, the modulation of the N200 component can be related to a distinct aspect of language processing.

\section{REFERENCES}

Bloom, P. A., \& Fischler, I. (1980). Completion norms for 329 sentence contexts. Memory and Cognition, 8, 631-642.

Brown, C. M., \& Hagoort, P. (1993). The processing nature of the N400: Evidence from masked priming. Journal of Cognitive Neuroscience, 5, 34-44.

Centre for Lexical Information (Producer and Distributor). (1990). CELEX Dutch database [Electronic database]. Nijmegen, The Netherlands: Centre for Lexical Information.

Connolly, J. F., \& Phillips, N. A. (1994). Event-related potential components reflect phonological and semantic processing of the terminal word of spoken sentences. Journal of Cognitive Neuroscience, 6, 256-266.

Connolly, J. F., Phillips, N. A., Stewart, S. H., \& Brake, W. G. (1992). Event-related potential sensitivity to acoustic and semantic properties of terminal words in sentences. Brain and Language, 43, 1-18.

Connolly, J. F., Stewart, S. H., \& Phillips, N. A. (1990). The effects of processing requirements on neuropsychological responses to spoken sentences. Brain and Language, 39, 302-318.

Goldinger, S. D., Luce, P. A., \& Pisoni, D. B. (1989). Priming lexical neighbours of spoken words: Effects of competition and inhibition. Journal of Memory and Language, 28, 501-518.

Goldinger, S. D., Luce, P. A., Pisoni, D. B., \& Marcario, J. K. (1992). Form-based priming in spoken word recognition: The roles of competition and bias. Journal of Experimental Psychology, Learning, Memory, and Cognition, 18, 12111238.

Grosjean, F. (1980). Spoken word recognition processes and the gating paradigm. Perception and Psychophysics, 28, 267-283.

Hagoort, P., \& Brown, C. M. (2000). ERP effects of listening to speech: Semantic ERP effects. Neuropsychologia, 38, 15181530 .

Holcomb, P. J. (1993). Semantic priming and stimulus degradation: Implications for the role of the N400 in language processing. Psychophysiology, 30, 47-61.

Juottonen, K., Revonsuo, A., \& Lang, H. (1996). Dissimilar age influences on two ERP waveforms (LPC and N400) reflecting semantic context effect. Cognitive Brain Research, 4, 99107.

Kutas, M., \& Federmeier, K. D. (2000). Electrophysiology reveals semantic memory use in language comprehension. Trends in Cognitive Sciences, 12, 463-470.

Kutas, M., \& Hillyard, S. A. (1980). Reading senseless sentences: Brain potentials reflect semantic incongruity. Science, 207, 203-205.

Kutas, M., \& Hillyard, S. A. (1984). Brain potentials during reading reflect word expectancy and semantic association. Nature, 307, 161-163.

Kutas, M., Lindamood, T., \& Hillyard, S. A. (1984). Word expectancy and event-related brain potentials during sentence processing. In S. Kornblum \& J. Requin (Eds.), Preparatory states and processes (pp. 217-238). Hillsdale, NJ: Erlbaum.

Kutas, M., \& Van Petten, C. (1988). Event-related brain potential studies of language. In P. K. Ackles, J. R. Jennings, \& M. G. H. Coles (Eds.), Advances in psychophysiology, vol. 3 (pp. 139-187). Greenwich, CT: JAI Press.

Kutas, M., \& Van Petten, C. (1994). Psycholinguistics electrified: Event-related brain potential investigations. In M. Gernsbacher (Ed.), Handbook of psycholinguistics (pp. 83-143). New York: Academic Press.

Marslen-Wilson, W. D. (1987). Functional parallelism in spoken word recognition. In U. H. Frauenfelder \& L. K. Tyler (Eds.), Spoken word recognition (pp. 71-102). Cambridge: MIT Press.

Marslen-Wilson, W. D., \& Tyler, L. K. (1980). The temporal structure of spoken language understanding. Cognition, 8, $1-71$.

Marslen-Wilson, W. D., \& Welsh, A. (1978). Processing interactions during word recognition in continuous speech. Cognitive Psychology, 10, 29-63.

Maxwell, S. E., \& Delaney, H. D. (1989). Designing and analyzing data (pp. 475-479). Pacific Grove, CA: Brooks/Cole.

McCarthy, G., \& Wood, C. C. (1985). Scalp distributions of event-related potentials: An ambiguity associated with analysis of variance models. Electroencephalography and Clinical Neurophysiology, 62, 203-208.

McClelland, J. L., \& Elman, J. L. (1986). The TRACE model of speech perception. Cognitive Psychology, 18, 1-86.

McQueen, J. M., Norris, D., \& Cutler, A. (1994). Competition in spoken word recognition: Spotting words in other words. Journal of Experimental Psychology, Learning, Memory, and Cognition, 20, 621-638.

Morton, J. (1969). Interaction of information in word perception. Psychological Review, 76, 165-178.

Näätänen, R. (1990). The role of attention in auditory information processing as revealed by event-related potentials. Behavioral and Brain Sciences, 13, 199-290.

Näätänen, R., \& Alho, K. (1997). Mismatch negativity-the measure for central sound representation accuracy. Audiology and Neuro-Otology, 2, 341-353.

Norris, D. (1994). Shortlist: A connectionist model of continuous speech recognition. Cognition, 52, 189-234.

Norris, D., McQueen, J. M., \& Cutler, A. (1995). Competition and segmentation in spoken word recognition. Journal of Experimental Psychology, Learning, Memory, and Cognition, 21, 1209-1228.

Osterhout, L., \& Holcomb, P. J. (1995). Event-related potentials 
and language comprehension. In M. D. Rugg \& M. G. H. Coles (Eds.), Electrophysiology of mind: Event-related brain potentials and cognition (pp. 171-215). New York: Oxford University Press.

Praamstra, P., Meyer, A. S., \& Levelt, W. J. M. (1994). Neurophysiological manifestations of phonological processing: Latency variation of a negative ERP component timelocked to phonological mismatch. Journal of Cognitive Neuroscience, 6, 204-219.

Pritchard, W. S., Shappell, S. A., \& Brandt, M. E. (1991). Psychophysiology of N200/N400: A review and classification scheme. In J. R. Jennings, P. K. Ackles, \& M. G. H. Coles (Eds.), Advances in psychophysiology, vol. 4 (pp. 43-106). Greenwich, CT: JAI Press.

Rösler, F., Heil, M., \& Glowolla, U. (1993). Monitoring retrieval from long-term memory by slow event-related brain potentials, Psychophysiology, 30, 170-182.
Salasoo, A., \& Pisoni, D. B. (1985). Interaction of knowledge sources in spoken word identification. Journal of Memory and Language, 24, 210-231.

Van Petten, C. (1995). Words and sentences: Event-related brain potential measures. Psychophysiology, 32, 511-525.

Van Petten, C., Coulson, S., Rubin, S., Plante, E., \& Parks, M. (1999). Time course of word identification and semantic integration in spoken language. Journal of Experimental Psychology, Learning, Memory, and Cognition, 25, 394-417.

Van Petten, C., \& Kutas, M. (1990). Interactions between sentence context and word frequency in event-related brain potentials. Memory and Cognition, 18, 380-393.

Zwitserlood, P. (1989). The locus of effects of sentential-semantic context in spoken-word processing. Cognition, 32, $25-64$. 